\title{
Contemporary Bulgaria. Political and Economic Perspectives after the European Adhesion
}

\author{
Roberto Sciarrone \\ Università di Roma La Sapienza \\ roberto.sciarrone@uniroma1.it
}

\section{Doi:10.5901/mjss.2013.v4n2p401}

\section{Abstract:}

The last decade has seen a relentless succession of economic crises, political and social factors that have distorted, in some cases, well-established diplomatic relations and development prospects, for better or for worse. These changes have had their greatest impact in the former socialist bloc, and particularly in the Balkans. Bulgaria, according to many studies, is part of this context and is equally conditioned by these considerations. The country, in fact, has gone through in recent years a strong political and social crisis which, with many difficulties, has led to a total economic revolution and a substantial progress in the integration into euro - Atlantic structures.

Keywords: Bulgaria, European Union, Socialism, Eastern Europe, European Community, Via Egnatia, European Treaties, Communist Party of Bulgaria, Zhivkov, Dimitrov of Bulgaria, Macedonia, Balkan Politics, Kosovo, Nikola Petkov.

\section{Preamble}

L'ultimo decennio ha visto un incessante susseguirsi di crisi economiche, politiche e sociali che hanno stravolto, in alcuni casi, rapporti diplomatici consolidati nel tempo e prospettive di sviluppo, nel bene o nel male. Questi cambiamenti hanno prodotto le loro conseguenze soprattutto negli Stati dell'ex blocco socialista(Biagini - Guida, 1994), e maggiormente nei Balcani (Biagini, 1990). La Bulgaria, paese che qui si tratta, non si ritiene, secondo numerosi studiosi, estranea a queste considerazioni. II paese, infatti, ha attraversato in questi ultimi anni una forte crisi politica e sociale che, con non poche difficoltà, ha portato ad una totale rivoluzione economica ed a un consistente avanzamento dell'integrazione nelle strutture euro - atlantiche. II $1^{\circ}$ gennaio 2007 la Bulgaria ha iniziato a far parte dell'Unione Europea (Bruno, 2012), dopo aver superato numerosi ostacoli, il paese è entrato in una nuova fase della sua storia in cui si troverà legato, come mai in passato, agli altri Stati europei. La forza di questo cambiamento epocale sul paese e i suoi abitanti sarà oggetto di studio per gli storici futuri (Dimitrov, 2011). La Bulgaria oggi è uno stato che conta circa 7 milioni di abitanti, ricco di gruppi etnici con prevalenza di bulgari, $83 \%$, e minoranze turche, $9 \%$, rom, $4 \%$ e altri. La superficie, $110.910 \mathrm{kmq}$, del paese confina con Grecia, Macedonia, Serbia, Romania e Turchia e comprende un territorio ricco di bauxite, carbone, rame e zinco. Bagnata dal Mar Nero la Bulgaria ha un tasso di crescita della popolazione dello 0,8\% ed un'aspettativa di vita media di 72,8 anni. La religione più praticata è quella cristiana ortodossa, $82 \%$, seguita da quella mussulmana, $12 \%$, e dalla cristiana protestante, cattolica romana, cristiana armena ed ebraismo. II tasso di disoccupazione, i questi ultimi anni, ha subito parecchie oscillazioni. Basti pensare che dal 13,5\% del 1997 si è rapidamente passati al 19,5\% del 2001, anno quest'ultimo caratterizzato da una forte crisi politica interna, per scendere nuovamente al 9,9\% nel 2005. Analizzando invece i dati relativi al PIL (in miliardi di dollari) dal 1997 al 2010 notiamo una lenta ma inesorabile crescita, un lieve aumento della produzione industriale e dei servizi ed una lieve diminuzione nel settore agricolo. Tutti questi dati ci servono, a mio parere, a comprendere meglio la complessa situazione economica, sociale e politica della Bulgaria all'indomani del crollo comunista (Giatzidis, 2002). Gli anni che vanno dal 1989 al 1997 possiamo definirli di "transizione incompleta" (Crampton, 2010). Tra il novembre 1989 e il dicembre 1990 si assiste allo smantellamento dell'apparato totalitario bulgaro. La caduta di Todor Hristov Zivkov (Bell, 1986), leader comunista per 35 anni, è decisa dal partito ed immediatamente dopo appaiono nel paese numerose organizzazioni politiche, alcune storiche come socialdemocratici e gli agrari, altre nuove (Aslanian, 2004). Gli "agrari", in particolar modo, si distinguono sin da subito dai collaborazionisti 
degli anni successivi al 1947 e riprendono il nome di Nikola Petkov': Unione nazionale agraria bulgara "Nikola Petkov"( BZNS - NP) (Padev, 1948). II 14 novembre 1989 ben 14 formazioni politiche, non comuniste, si organizzano quindi in una federazione che prende il nome di "Unione delle forze democratiche" (SDS), la guida è assunta da Zelju Zelev, professore universitario di filosofia. II nuovo leader del Partito comunista bulgaro (BKP) (Isusov, 1975), Petar Mladenov, riorganizza i quadri e medita sugli errori del passato. Ciò non basta a calmare le acque politiche del paese, lo SDS organizza a Sofia una manifestazione per chiedere l'abolizione del primo articolo della Costituzione, articolo che garantiva al BKP un ruolo di guida nella società bulgara. Mladenov ed i suoi seguaci aprono quindi ad una trattativa che si preannuncerà serrata e difficile, sotto molti punti di vista, con le forze democratiche. In ballo c'è il futuro della Bulgaria. Le proteste intanto continuano copiose e invadono tutto il Paese ma soprattutto la capitale. II 7 gennaio migliaia di dimostranti arrivano a Sofia da tutta la nazione: tutti chiedono di smantellare l'apparato totalitario e di trovare una soluzione per eludere l'articolo 1 della Costituzione che, di fatto, bloccava le nuove forze politiche emergenti. II comitato alla fine, decide per l'emendare l'articolo 1 con una scelta dettata più dagli eventi che da una lucida idea politica (Crampton, 2010). Poco tempo dopo viene abolito anche il vecchio consiglio centrale dei sindacati, l'organizzazione della gioventù comunista e il fronte patriottico (OF). Alla fine di gennaio il Partito comunista bulgaro convoca il XIV congresso risolvendosi per una netta riorganizzazione. II politbjuro e il comitato centrale vengono sostituiti da organi più rappresentativi e viene annunciato l'arresto di Zivkov incriminato per abuso di potere ed incitamento all'odio razziale (Mclntryre, 1988). Mladenov dichiara l'intenzione di voler riformare l'economia secondo le regole della privatizzazione, della decentralizzazione e della de - monopolizzazione, una democrazia multipartitica e la volontà di giungere a una completa separazione tra Stato e partito. Dopo queste affermazioni Mladenov lascia l'incarico di segretario generale del BKP, che passa a Aleksandar Lilov, mantenendo per sé la carica di capo dello Stato e forma un nuovo governo nazionale con Andrej Lukanov premier. II gesto forse più sintomatico della nuova ondata di cambiamenti è stato senza dubbio lo scioglimento della polizia segreta bulgara, nella fattispecie il VI dipartimento del ministero degli Interni, senza dimenticare l'agognato riconoscimento del diritto allo sciopero. Le elezioni del giugno 1990 assegnano 211 seggi al BSP, 114 allo SDS, 23 al Movimento per i diritti e la libertà (DPS), principale gruppo politico della minoranza turca. Lukanov è riconfermato premier. Pochi giorni dopo le elezioni i contestatori rendono però pubblico un filmato che, come dichiarano, mostra Mladenov che richiede l'invio di mezzi corazzati per fermare le manifestazioni del dicembre 1989, scoppia una polemica dai toni accesi. Mladenov rassegna le dimissioni da presidente e viene immediatamente sostituito da Zelev, uno zoologo di professione, Petar Beron, assume la carica di capo dello SDS. Le polemiche non si fermano e continuano incessanti attraverso numerose dimostrazioni di piazza per tutto il 1990. II 29 novembre arrivano anche le dimissioni di Lukanov e il 20 dicembre viene formato un nuovo governo nazionale con a capo un avvocato: Dimitar Popop.

Tra il novembre 1989 e il dicembre 1990 la gran parte degli organi del sistema totalitario è stata smantellata. La Bulgaria può così presentarsi al mondo esterno sotto una nuova veste. Gli stretti legami con l'URSS hanno lasciato il posto a nuovi rapporti diplomatici con Israele, Cile e Sudafrica. Unica fase da completare: l'insediamento di un regime democratico vero (Brown, 1970). Poche riforme infatti, hanno sin qui garantito un facile trapasso dal regime comunista al nuovo. Popop capisce che per risollevare le sorti del proprio Paese c'è bisogno di perseguire la via del dialogo tra le numerose e discordanti forze politiche bulgare. L'esecutivo stesso si autodefinisce "un governo per garantire la transizione pacifica a una società democratica"(Crampton, 2010): La priorità, come accaduto nei governi successivi alla fine del comunismo, è affidata all'economia. L'8 gennaio 1991 viene firmato tra i datori di lavoro e i sindacati, assieme al governo, un accordo tripartito per la pace sociale. I sindacati accettano una moratoria di 200 giorni sugli scioperi. La pace sociale è messa subito alla prova quando il governo Popop opta, seguendo l'esempio polacco, per rimedi economici aggressivi aventi come principio cardine la deregolamentazione dei prezzi di numerosi prodotti. Oltre alle pesante ricadute sociali di tali decisioni iniziano con una certa regolarità a manifestarsi violente tensioni di carattere etnico. A febbraio il ministero dell'Istruzione annuncia che nelle regioni (Biagini, 2005)abitate dai turchi sarebbe stato possibile inserire nelle scuole quattro ore a settimana di insegnamento della lingua di minoranza (Biagini, 1981). Tale annuncio causa una serie di proteste dai toni molto forti e si assiste allo sciopero dei docenti delle zone coinvolte (Karpat, 1990).

${ }^{1}$ Nikola Petkov ( Sofia, 8 luglio 1893 - Sofia, 23 settembre 1947) è stato un politico bulgaro. Nel 1936 fu eletto deputato del Partito agrario e si batté contro la dittatura di re Boris III e successivamente contro la sua politica tendente all'alleanza con la Germania nazista e l'Italia fascista. Esponente dell'ala sinistra del Partito agrario e capo dei Pladnenzi, fu arrestato nel 1941. Dopo essere stato liberato, fu tra i promotori del Fronte Patriottico, che nel settembre 1944 assunse il potere. Divenne vicepresidente del Consiglio nel primo governo Georgiev e firmò l'armistizio con l'Unione Sovietica. Dopo aver mantenuto la coalizione con i comunisti, fini per rompere con loro e dare le dimissioni. Passò all'opposizione, dirigendo la Nuova bandiera contadina, ma il 6 giugno $1947 \mathrm{fu}$ arrestato e poi processato con l'imputazione di complotto contro lo Stato. Condannato a morte il 15 agosto, si vede respingere l'appello il 18 settembre, nonostante le numerose proteste di americani e di altri esponenti occidentali. Venne impiccato a Sofia cinque giorni dopo. 
Dopo ulteriori vicissitudini arriva finalmente la convocazione della Grande assemblea nazionale che si dedica alla definizione della struttura politica del Paese e il 12 luglio è approvata la nuova Costituzione. La Bulgaria diventa uno Stato democratico ispirato al principio della legalità e alla separazione dei poteri. II capo dello Stato avrebbe dovuto essere eletto con suffragio diretto e rimanere in carica cinque anni, inoltre tutti i candidati alla prestigiosa carica dovevano essere residenti in Bulgaria da almeno cinque anni. Quest'ultima norma è chiaramente studiata dall'assemblea costituente per evitare che re Simeone potesse concorrere alle presidenziali. E' istituita anche una corte costituzionale è si decide che, per ogni legislatura, sarebbe stato nominato un parlamento di durata quadriennale composto da 240 deputati scelti col sistema proporzionale e con una soglia di sbarramento per i partiti fissata al $4 \%$ del totale dei voti su scala nazionale. Le prime elezioni con il nuovo sistema si svolgono il 13 ottobre 1991 ed alla votazione partecipano ben 38 partiti, solo 3 di questi supereranno lo sbarramento del $4 \%$ ad ogni modo, il risultato, esprime un equilibrio tale da creare sin dai primi giorni successivi al voto motivo d'allarme. Lo SDS si assicura la maggioranza relativa con una percentuale di voto superiore di solo l'1\% a quella del BSP e ottiene 110 seggi, contro i 106 dell'opposizione. II DPS, con i suoi 24 deputati, diventa l'ago della bilancia. Più del $20 \%$ delle preferenze va a partiti che non raggiungono la soglia del 4\%. II nuovo governo, con alla guida il leader dello SDS Filip Dimitrov, dura poco più di un anno anche per la rinuncia del DPS di aderire alla coalizione appena formata. Dimitrov dimostra sin da subito voglia di rinnovare gli apparati statali e dare nuovo impulso all'economia bulgara ma i risultati che conseguirà non saranno all'altezza delle pressanti aspettative, l'opinione pubblica criticherà costantemente il nuovo esecutivo soprattutto sul tema "epurazione". Dimitrov accelera il processo di rassegnazione delle terre confiscate ai comunisti e quello di epurazione, circa 750 funzionari del vecchio regime sono mandati in pensione. L'impegno in tal senso si riconferma in settembre con la condanna di Zivkov a sette anni di reclusione, primo fra gli ex leader dell'Europa orientale a essere processato e condannato, anche se riuscirà a evitare il carcere grazie alla concessione degli arresti domiciliari (Trinchese, 2005). Dalle università sono estromessi tutti coloro che avevano detenuto cariche nel partito sotto il vecchio regime (Kostov, 1988), legge Panev. II sistema delle epurazioni nella Chiesa ortodossa bulgara (Graciotti, 2007), si risolve però in farsa e tragedia. II 9 marzo 1992, infatti, il patriarca Maksim è rimosso e accusato di collaborazionismo e di elezione, avvenuta nel 1971, irregolare. La Corte costituzionale prende spunto dalla vicenda per affermare l'indebita intrusione dello Stato su questioni "prettamente ecclesiastiche". Molti rifiutano di accettare la sentenza e occupano la sede del Santo sinodo e la Chiesa ortodossa, insomma una situazione disarmante per una nazione che aspira ad entrare in Europa. In mezzo ai numerosi fallimenti di politica interna, il governo Dimitrov raccoglie discrete soddisfazioni in politica estera come l'ammissione della Bulgaria al Consiglio d'Europa ${ }^{2}$, maggio 1992. Migliorano i rapporti con Ankara (Simsir, 1985), in particolar modo sulle linee da seguire rispetto alla crisi jugoslava e le sue conseguenze. La Bulgaria, dopo la dissoluzione del Patto di Varsavia (Gokay, 2005), ritiene di dover costruire un nuovo sistema di forze e protezione dagli Stati confinanti, il più grande dei quali è la Turchia e nel maggio 1992 viene siglato un trattato di amicizia tra i due Paesi. Una delle questioni più scottanti è però legata, in quel periodo, alla Macedonia (Bozzo, 2000), in quanto pochi giorni dopo il 10 novembre 1989 gli abitanti della Bulgaria che si consideravano macedoni pensarono di fondare un loro partito (Brailsford, 1986). La formazione non ottiene però alcun riconoscimento da parte degli organi competenti bulgari e dopo la fine della Federazione jugoslava (Perich, 1998) e la proclamazione di indipendenza del governo di Skopje il governo di Sofia è in seria difficoltà. Ad ogni modo la decisione della Bulgaria di riconoscere lo Stato macedone, il 16 gennaio 1992, rimane nell'immediato una scelta controversa (Barker, 1950). Ad esempio il ministro degli Esteri non viene avvisato, alcuni Paesi come la Grecia rimangono colpiti dalla linea politica di Sofia. Atene ad esempio rifiutava il riconoscimento di qualsiasi Stato che portasse il nome "Macedonia" (Perich, 2004). La Bulgaria, d'altro canto, non può permettersi, nel delicato frangente politico che sta ricomponendo, di mandare in crisi i suoi rapporti con la vicina Grecia, importante partner commerciale. II governo di Sofia trova, quindi, un compromesso per non alterare i faticosi equilibri della zona e con decisione riconosce lo Stato ma non l'esistenza di una distinta nazione macedone. Sofia inoltre nega l'esistenza di una distinta lingua macedone, che i governati di Skopje volevano impiegare nella stesura degli accordi e nei rapporti ufficiali tra i due Stati. I rapporti tra le due istituzioni rimangono pessimi. In politica interna le riforme di carattere economico hanno colpito le regioni turche in misura maggiore rispetto al resto del Paese: la risposta è l'emigrazione. Se questa ondata di espatri fosse continuata, la popolazione turca si sarebbe talmente ridotta da privare il DPS della percentuale di voto del $4 \%$ necessaria per superare lo sbarramento elettorale. In settembre il Movimento per i diritti e la libertà spinge il presidente del parlamento a rassegnare le dimissioni. II governo Dimitrov non si dimostra quindi all'altezza delle aspettative dei suoi sostenitori, senza

\footnotetext{
2 Per comprendere i cambiamenti istituzionali dell'UE in vigore dal 1 gennaio 2007 con l'adesione della Romania e della Bulgaria, numero dei parlamentari europei, modalità di votazione in Consiglio, composizione di altri organi, ecc., e conoscere la nuova struttura di alcune politiche comunitarie (agricoltura e pesca, coesione economica e sociale, ricerca e sviluppo) e i numerosi programmi avviati nel 2007 in seguito all'adozione della programmazione finanziaria valida fino al 2013.
} 
contare il mancato raggiungimento del giusto bilanciamento di poteri tra l'esecutivo e la presidenza (Moser, 1989). II vuoto che lascia il governo Dimitrov non può, ad ogni modo, essere colmato dal BSP, poiché non dispone del necessario consenso parlamentare né un leader con autorità già consolidata all'interno del partito. II presidente e il DPS si presentano come le uniche due forze, quest'ultima da il suo consenso all'appoggio esterno per un governo tecnico formato da esponenti pubblici apartitici. L'incarico di primo ministro va a Ljuben Berov, docente di storia economica, già consulente finanziario del presidente. In politica estera grande ottimismo è generato da una dichiarazione dell'Unione europea (UE) in relazione ad un accordo commerciale temporaneo con Sofia. Ottimismo subito stroncato dalla decisione europea di rimandare l'applicazione degli accordi sino al dicembre 1993. II nuovo premier Berov cerca di resistere alla crisi economica che attanaglia il Paese in quegli anni, ma tutti i tentativi di riprendere le riforme economiche, adeguandole e ritoccandole, falliscono e alimentano tensioni sociali e il continuo malumore dell'opinione pubblica. Uno dei problemi principali, come ricorda lo storico Richard J. Crampton, è: "la mancanza di un chiaro sostegno parlamentare, l'assenza di un'amministrazione stabile si espresse soprattutto nella costante incapacità di stilare un adeguato programma di riforme economiche e di contrastare l'aumento della criminalità, con l'imposizione delle sanzioni contro la Jugoslavia i due problemi si acuirono" (Crampton, 2010). II governo Berov è subito criticato dall'opinione pubblica, molti i problemi ancora da risolvere tra cui la crisi economica e l'aumento del crimine, termina nel settembre del 1994 ed è sostituito da un'amministrazione temporanea con alla guida la prima donna primo ministro di Bulgaria: Reneta Indzova. Alle elezioni di dicembre il BSP ottiene una buona vittoria. II falliemento del precedente governo è da addebitare alla scarsa incisività nel contrastare criminalità e debolezza economica e l'affermazione dei socialisti in parte è dovuto a questi fattori, ma non solo. I cinque anni precedenti erano stati interpretati come una fase di egemonia dello SDS, che veniva accusato di non essere stato in grado di attuare delle corrette riforme in campo economico e di non aver saputo contrastare nella giusta maniera la dilagante corruzione che dilaniava il Paese. La ricostruzione politica, in questi primi anni "post '89", ha maggiore rilievo rispetto le riforme economiche. Analizziamo adesso i primi cinque anni di riforme economiche, molte delle quali inespresse. Nel 1989, durante un discorso al plenum del comitato centrale, Mladenov aveva ammesso che il debito estero della Bulgaria non ammontava a tre miliardi di dollari bensì a dodici. Queste condizioni economiche rendono quasi impossibile per la Bulgaria pensare in maniera ottimistica rispetto ad accordi commerciali con l'UE e l'area dollaro. Inoltre numerosi fattori di ragione pratica rendono meno spendibili sui mercati esteri i prodotti bulgari, poco "appeal" insomma. La vendita all'estero di prodotti di base si presenta complicata, anche perché ciò che Sofia può offrire è rappresentato da merci, come vino e frutta, che l'UE già produce in quantità considerevole. II collasso del COMECON (Leonardi, 1977) aveva determinato questa crisi economica strutturale della Bulgaria, che cerca di mirare obiettivi prestigiosi, come l'ingresso nell'area dell'UE, senza riuscire concretamente a trovare gli strumenti più adatti. Inoltre vengono ridotti gli scambi con due dei più importanti precedenti partner economici, cioè la Libia e l'Iraq, per salvaguardare l'immagine del Paese nei confronti dell'Unione Europea che adottava sanzioni rigorose nei riguardi dei due Stati. Questa scelta politica ha quindi forti ripercussioni, come detto, poiché l'Iraq rappresenta il principale paese debitore della Bulgaria e aveva da poco firmato un accordo in base al quale il disavanzo sarebbe stato ripagato in petrolio. Nonostante tutti i problemi economici i paesi occidentali, ma soprattutto gli Stati Uniti, sono ancora disposti ad aiutare la Bulgaria. Lukanov, per un lungo periodo, viene assistito da consulenti statunitensi per la stesura del programma di riforme urgenti. Nei primi sette mesi del 1990 la produzione scende del 10\% e l'inflazione tocca quota $108 \%$. Lukanov non era riuscito a rimediare alla situazione. II governo Popov tenta di porre rimedio scatenando il "big bang" della Bulgaria il $1^{\circ}$ febbraio 1991. In quel periodo si assiste ad un acceso dibattito parlamentare al termine del quale il governo riesce a far approvare delle leggi che aprono la via alla futura de collettivizzazione dell'agricoltura e alla privatizzazione delle piccole imprese. In estate una seconda tranche di riforme introduce una liberalizzazione dei prezzi e impone alla Banca nazionale bulgara l'obbligo di rispondere alle sue azioni davanti al parlamento, non solo al governo. II governo Dimitrov, come scritto in precedenza, prosegue con le riforme strutturali e nel febbraio 1992 vengono legalizzate la proprietà straniera delle imprese bulgare e l'esportazione dei profitti, più numerose norme di carattere bancario. Pochi giorni dopo esser entrato in carica il primo ministro Berov presenta invece un piano d'azione economico che non ha però molto successo. Gli investimenti interni sono scarsi e insufficienti e le privatizzazioni, quelle delle terre su tutte, procedono con molta lentezza. Nell'estate del 1994 il valore del lev ${ }^{3}$ inizia a scendere e gli interventi di sostegno della Banca nazionale bulgara determinano l'aumento inesorabile dell'inflazione.

3 II Lev è la valuta ufficiale della Bulgaria, usata dal 1881. E' diviso in 100 stotinki. Negli anni '90 il valore del lev è stato agganciato a quello del Marco tedesco. II 5 luglio 1999 la Banca centrale bulgara ha deciso di rivalutare il lev introducendo il nuovo Lev con il seguente cambio rispetto al vecchio: 1000 vecchi Lev=1 nuovo Lev. Oggi, settembre 2012, il cambio del Lev è fissato con l'euro con il valore di 1,95583 Lev = 1 EUR. 
Inoltre, per ragioni di natura politica, la Bulgaria aggrava la situazione accettando di rispettare le sanzioni applicate alla Serbia e al Montenegro nel 1992, aumentate nella primavera del 1993 (Ramet, 2009). Sofia si attiene quindi alla linea ONU ma, come abbiamo visto in precedenza riguardo le sanzioni imposte all'lraq, le conseguenze per il paese furono molto gravi. Le ferrovie e le strade dirette verso l'ex Jugoslavia costituivano infatti uno degli assi principali del commercio bulgaro, le sole alternative disponibili, attraverso la Romania o via mare, sono lente e congestionate. In settembre il costo delle sanzioni è stimato a 2, 71 miliardi di dollari, sommando anche quelle dirette contro Libia e Iraq si arriva a 13 miliardi ovvero l'intero debito estero del paese. II risultato delle elezioni del 1996 assegna al BSP la prima maggioranza parlamentare assoluta ottenuta da un partito dopo il 1989. Ai socialisti vanno il $52 \%$ dei voti e 125 seggi, allo SDS il $28 \%$ e 69 seggi e al DPS il 6\% e 15 seggi. Entrano in parlamento anche l'Unione popolare (NS) e il Blocco degli imprenditori bulgari (BBP) che superano lo sbarramento del 4\%. II ritorno di un partito forte con alle spalle una maggioranza parlamentare assoluta suscita nell'opinione pubblica bulgara speranze di rinnovamento. All'inizio del suo mandato Zhan Videnov mantiene le attese. I suoi obiettivi sono l'inversione del declino economico, consolidare l'integrazione della Bulgaria nelle istituzioni europee, lotta al crimine e la ripresa delle relazioni estere con la Russia. Nessuno degli obiettivi annunciati da Videnov viene raggiunto. La Bulgaria è sull'orlo del baratro economico e sociale. I primi passi del neo governo, del resto, infondevano fiducia ad un ambiente politico depresso. I rapporti con Mosca, ad esempio, già risanati con Berov, si fanno ancora più stretti nel maggio 1995 grazie agli accordi sulla cooperazione commerciale, la difesa e la costruzione di gasdotti e oleodotti per il trasporto del metano e del petrolio russi dal porto bulgaro di Burgas sino ad Alessandropoli, in Grecia, ed altre località situate nei Balcani (D'Amato, 2008). Per perfezionare l'accordo viene creata una compagnia mista russo - bulgara, la Top - Energy, alla cui guida viene nominato l'ex primo ministro Lukanov. Anche il settore economico sembra rinascere, nel 1995 il Pil sale del 3\% e l'inflazione passa dal 59\% al 15,2\%. La Banca nazionale abbassa i tassi d'interesse non meno di sette volte nel 1995. La ripresa economica si concretizza in un'illusione ed anche le relazioni stabilite con Mosca pochi mesi prima iniziano a contaminarsi. Durante le trattative per la costruzione degli oleodotti e gasdotti i russi si dimostrano inflessibili circa i termini degli accordi lasciando poco margine di manovra a Sofia. Inoltre Mosca pretende dal governo di Sofia rassicurazioni circa l'impegno di quest'ultimo a non entrare nella NATO. Alla fine del 1996 le relazioni tra i due paesi sono peggiori rispetto a quando Videnov era entrato in carica. II fallimento più grave del governo Videnov riguarda però più la politica interna che quella estera. L'emergenza alimentare inizia a premere sulla debole economia bulgara, scelte sbagliate, come l'esportazione del grano all'estero durante i numerosi periodi di carestia, determinano lo sconforto della popolazione. La finanza del paese non gode di buona salute, sui mercati valutari il lev va sotto pressione. II crimine aumenta, disattendendo le promesse fatte di Videnov durante il discorso d'insediamento. La mafia bulgara si oppone costantemente alla ristrutturazione economica del paese di fatto rallentando ulteriormente la crescita. I| 16 maggio è la volta del "venerdì nero", con due istituti di credito che vengono sottoposti all'amministrazione controllata della Banca nazionale. II governo, costretto ad agire, annuncia che 64 fra le più grandi imprese in perdita sarebbero state chiuse e altre 70 poste in isolamento, ovvero private di ulteriori prestiti bancari. E' la catastrofe annunciata. II lev intanto continua la sua inesorabile discesa e l'FMI annuncia la sospensione degli aiuti fino a che il governo di Sofia non gli avesse concesso voce in capitolo all'interno della Banca nazionale 4 . II rifiuto netto del governo di accettare questi compromessi causa un'ulteriore perdita di fiducia nelle banche, nove delle quali sono state, nel frattempo, poste sotto il controllo dell'istituto bancario nazionale in settembre, i tassi d'interesse toccano il $300 \%$. L'inflazione, nel 1996, non accenna a diminuire e tocca il 578,6\%. La popolazione non assiste inerte all'incedere della crisi, anzi organizza grandi manifestazioni nella capitale una delle quali, il 7 giugno, vede circa 1 milione di persone protestare alacremente. Durante le elezioni presidenziali di ottobre - novembre emerge tutto il malcontento della popolazione. II nuovo candidato dello SDS, un avvocato anticomunista, Petar Stojanov, vince al ballottaggio e ottiene il $60 \%$ dei voti contro il $40 \%$ del candidato del BPS. Pochi giorni dopo, il 21 dicembre, Vedinov si dimette sia dalla carica di capo del governo, che da quella di leader del suo partito. Al termine del 1996, la Bulgaria vede svanire sempre più la debole possibilità che il proprio Paese venga incluso nella lista di Stati che chiedevano di aderire all'UE (Lampe, 1999). Nel gennaio 1997 il panorama politico inizia a mutare. Lo SDS di Ivan Kostov, ex ministro delle finanze del governo Dimitrov, annuncia che, al contrario del BSP, è pronto a seguire i dettami dell'FMl e a introdurre una commissione per il controllo della valuta. La situazione sembra rasserenarsi quando all'improvviso, il 10 gennaio, l'atmosfera torna rovente a causa della decisione di lasciare il parlamento da parte degli onorevoli dell'opposizione. Nel Paese tornano gli scioperi e

4/l Fondo monetario internazionale da più di sessant'anni è, insieme al Wto e alla Banca mondiale - le altre due organizzazioni nate dagli accordi di Bretton Woods nel 1944 - un pilastro dell'ordine economico internazionale. Ne sono prova gli interventi messi in atto dal Fondo monetario in molti Paesi, come la Bulgaria, per fronteggiare la crisi dei mutui subprime, e il sostegno fornito alla Grecia, che nel maggio 2010 ha rischiato la bancarotta. Nel volume si spiega che cosa è e come funziona il Fondo monetario internazionale, qual è il ruolo che ha svolto finora e come si è trasformato nell'età della globalizzazione fino alle ultime crisi finanziarie. 
la rivoluzione sembra vicina come non mai dal 1989 ( Dahrendorf, 1999). Intanto al governo Kostov succede un nuovo esecutivo formato dal leader del partito di maggioranza Dobrev che in extremis riesce a sedare le proteste annunciando nuove elezioni in aprile e affidando la guida provvisoria del Paese, l'ennesima in pochi mesi, al sindaco di Sofia, Stefan Sofijanski. Lo SDS intanto, si unisce, in posizione di maggioranza, al piccolo gruppo di centro - destra dell'NS e si presenta alle elezioni di aprile sotto la sigla ODS, Forze democratiche unite. I risultati, molto prevedibili, vedono la netta vittoria del nuovo gruppo con il 52\% dei voti e 137 seggi nel sabranie, il BSP si arresta al $22 \%$ e 58 seggi. L'unica alternativa valida per uscire dalla crisi sarebbe stata ora quella di cercare rifugio e protezione all'interno delle strutture euroatlantiche, in modo particolare la NATO e l'UE. A partire dal 1997 inizia così un processo di ricerca della stabilità che durerà fino al 2004, a fasi alterne. II neo governo Kostov riesce nell'impresa di concludere il proprio mandato quadriennale, piccolo record nella storia della Bulgaria postcomunista. Un altro successo, non meno importante ma di natura diversa, viene conseguito nel settembre del 1999, quando la conferenza di Helsinki5 dell'Unione Europea non si oppone all'inserimento della Bulgaria nella lista degli Stati che avrebbero potuto negoziare la loro entrata nell'organizzazione. Nel marzo del 2000 è concesso al Paese di avviare le trattative in relazione a 8 dei 31 punti dell'acquis comunitario. I progressi sulla strada dell'inserimento dipendono in primis dalla stabilizzazione economica, dal risanamento e dalle riforme strutturali, senza dimenticare i rigidi paletti imposti sul terreno del contenimento del crimine e della corruzione. La stabilizzazione economica viene comunque raggiunta in tempi brevi. II Lev viene agganciato al marco tedesco, dal 1999 all'euro, e alla Banca nazionale è proibita l'introduzione di liquidità, la cura, insomma, si rivela efficace. L'inflazione ad esempio, schizzata al $247 \%$ nel febbraio 1997, nel luglio del 1999 cala all'1,7\%, mentre il debito estero subisce una drastica diminuzione. La ritrovata stabilità finanziaria favorisce la ripresa dei salari dall'erosione inflattiva del 1996 e del 1997, la media degli stipendi mensili dei dipendenti pubblici, nel febbraio del 1997 era di soli 25 dollari, nel maggio 1999 sale a 124. Inoltre nel gennaio parte una nuova politica di privatizzazioni, vengono messe in vendita 31 società, in un anno circa il $70 \%$ delle imprese bulgare non sarebbe più stato proprietà dello Stato. Un'altra vittoria in campo economico è la liquidazione di numerose aziende statali in perdita infatti, nel luglio '99, il ministro delle Finanze annuncia che la Bulgaria aveva accettato la scadenza imposta dall'FMl per la chiusura o la vendita di 41 grandi imprese con bilanci in passivo, come il grande complesso metallurgico di Kremikovci il cui prezzo di vendita viene fissato ad un dollaro. Ad ogni modo gli sforzi economici del Paese non passano inosservati e ricevono le lodi delle istituzioni finanziare internazionali. La buona volontà di Sofia è premiata attraverso la concessione di sovvenzioni di vario titolo come 125 milioni di dollari accordati dall'Unione Europea e un prestito incondizionato di 200 milioni di dollari dalla Banca mondiale ${ }^{6}$.

\section{The New Century}

Nel 2000 la Bulgaria chiude 311 stabilimenti di lavorazione della carne e 230 caseifici, poiché non rispondenti alle direttive comunitarie ed abolisce i dazi sull'importazione di 470 prodotti agricoli made in UE. Le richieste dell'Unione Europea non si limitano al settore economico, altre riforme del governo Kostov si legheranno infatti a espliciti desideri di Bruxelles. Sempre nel 2000 è avviata la trasmissione di programmi in lingua turca da parte delle emittenti ufficiali e un anno dopo, gennaio 2001, una riforma dell'istruzione, mirata ad allargare le prospettive di entrata nelle istituzioni

5/l Consiglio europeo di Helsinki (10 e 11 dicembre 1999) ha confermato l'importanza delprocesso di ampliamento, in particolare al fine di garantire la stabilità e la prosperità inEuropa. Ha inoltre riaffermato il carattere inclusivo del processo, al quale $i 13$ paesicandidati partecipano su un piede d'uguaglianza. A questo proposito, il Consiglio europeoha sottolineato che la Turchia è un paese candidato.ll Consiglio europeo di Helsinki ha posto l'accento sulla necessità per i Paesi candidati dicondividere $i$ valori e gli obiettivi dell'Unione europea enunciati nei trattati. Ha invitato iPaesi candidati a risolvere le eventuali controversie relative alle frontiere (e altre questioni)conformemente ai principi della Carta delle Nazioni Unite o, altrimenti, a deferire lecontroversie alla Corte internazionale di giustizia entro un termine ragionevole. Inoltre, il Consiglio europeo di Helsinki ha ricordato che il rispetto dei criteri politici fissatidal Consiglio europeo di Copenaghen rappresenta una condizione preliminare per l'avviodei negoziati di adesione.ll Consiglio europeo ha deciso di organizzare, nel febbraio 2000, alcune conferenzeintergovernative bilaterali in vista di avviare negoziati con la Romania, la Slovacchia, laLettonia, la Lituania, la Bulgaria e Malta sulle condizioni di adesione all'Unione europea esugli adattamenti che occorrerà di conseguenza apportare ai trattati. Ha altresi preso attodella nuova valutazione effettuata dalla Commissione riguardo ai progressi ottenuti daiPaesi candidati (relazioni del 13 ottobre 1999). La valutazione, pur constatando che sonostati ottenuti alcuni progressi, ha messo in evidenza che taluni Paesi candidati nonsaranno in grado di rispettare a medio termine tutti $i$ criteri di Copenaghen.

${ }^{6}$ La Banca Mondiale, BM, comprende due istituzioni internazionali: la Banca Internazionale per la Ricostruzione e lo Sviluppo, BIRS, e l'Agenzia Internazionale per lo sviluppo, AID, create per lottare contro la povertà e per organizzare aiuti e finanziamenti agli Stati in difficoltà. La sua sede è a Washington D.C. ed il suo presidente è eletto per cinque anni dal consiglio d'amministrazione della banca. La BM è stata creata il 27 dicembre 1945, dopo la firma dell'accordo di Bretton Woods. 
europee, sono introdotti corsi di integrazione all'Unione Europea nelle scuole secondarie. Gli studenti avrebbero infatti studiato l'Europa contemporanea con lezioni di storia, geografia, economia e filosofia. Le novità non finiscono qui, i registri della polizia sono resi pubblici, insieme ai nomi di numerosi politici e funzionari statali che avevano servito gli apparati di spionaggio comunisti. Se la riforma economica, l'allargamento dell'istruzione nella lingua della minoranza e una maggiore trasparenza nella vita nazionale erano stati voluti dal governo Kostov, da Bruxelles giungono altre richieste la cui attuazione si rivela più problematica da perseguire. Tre le richieste principali: chiusura di reparti del complesso nucleare di Kozloduj, la riforma dell'apparato giudiziario e la lotta alla corruzione. L'Unione Europea ritiene, nel 1999, insicuri quattro dei sei reattori di Kozloduj e ne reclama la chiusura. L'impianto all'epoca produceva un terzo dell'energia della Bulgaria e nel Paese non esistevano altre fonti alternative. La richiesta, come si vede, è di difficile comprensione da parte dei politici bulgari che tuttavia, nella figura del primo ministro Kostov, cedono alle richieste europee. Dal 1999 al 2006 vengono chiusi i reattori incriminati, in cambio l'UE promette 200 milioni di dollari per ammortizzare gli effetti della disattivazione. II grave problema della riforma giudiziaria non è mai affrontato in maniera sinergica dal governo di Sofia, almeno sotto la guida Kostov. La questione è strettamente legata alla corruzione contro la quale l'Europa che conta pone e invoca più volte severi provvedimenti. Per ciò che concerne la politica estera il governo bulgaro fornisce pieno appoggio diplomatico all'intervento NATO in Kosovo e concede l'uso dello spazio aereo all'aviazione dell'alleanza, privilegio negato ai russi (Arielli, 1999). La decisione è premiata con la visita del presidente Bill Clinton a Sofia, primo presidente degli Stati Uniti a recarsi in Bulgaria (White, 2012). Parallelamente a decisioni di politica estera compiacenti alle istituzioni internazionali occidentali Sofia avvia la ristrutturazione del suo apparato militare, ormai vetusto. Nel settembre 1999 è annunciato il "Piano 2004" di riduzione e miglioramento dell'efficienza della forza militare. Un altro passo verso la NATO riguarda la creazione, molto apprezzata dall'organizzazione nord -atlantica, di una forza di pace regionale balcanica. I ministri della Difesa, infatti, di Italia, Albania, Bulgaria, Grecia, Macedonia, Romania e Turchia approvano nel settembre 1998 l'istituzione di un corpo comune di 3mila uomini, con comando a Plovdiv per i primi 4 anni ed assegnato a rotazione successivamente. II veto posto dalla NATO all'ingresso nell'alleanza di membri che avevano ancora dispute relative ai confini con i propri vicini è alla base dell'accordo concluso tra Bulgaria e Macedonia nel febbraio '99. I due Stati ritirano ogni rivendicazione sul territorio altrui e risolvono infine l'annoso problema della lingua da impiegare per redigere il verbale d'intesa, che viene quindi sottoscritto nelle lingue ufficialmente in uso in ciascuno dei due Paesi. La Bulgaria, così facendo, riconosce l'esistenza di una lingua macedone senza altresì dichiararlo in maniera esplicita. Le relazioni tra Bulgaria e Macedonia miglioreranno in maniera graduale grazie a questi importanti accordi. Sempre durante il governo Kostov un altro passo avanti, nelle relazioni tra Stati nei Balcani, è la stipula di un'intesa con la Romania per la costruzione di un secondo ponte sul fiume Danubio. Durante le prime fasi della trattativa Bucarest si dimostra contraria alla costruzione del ponte nel settore occidentale della frontiera tra i due paesi, i romeni infatti preferiscono una direttrice orientale, più vicina alla capitale, due i fattori che fanno cambiare idea al governo romeno: in primis le pressioni di Bruxelles che vuole inserire il ponte all'interno del corridoio pan - europeo di transito 4, progetto di collegamento viario tra Grecia ed Europa occidentale attraverso Bulgaria, Romania e Ungheria (Biagini, 2006) e la già citata crisi del Kosovo ${ }^{7}$ che rivela la scarsa rete di comunicazioni dell'area in mancanza di vie d'accesso jugoslave. L'entrata dei cittadini bulgari nell'area Schengen raccoglie inoltre numerosi consensi, l'accordo, entrato in vigore nell'aprile 2001, consente finalmente ai bulgari di potersi recare in quasi tutti gli Stati dell'UE senza visti per la cui concessione passavano anche mesi. II governo Kostov cerca di mantenere alta la sua popolarità attraverso questi progressi di carattere internazionale, poiché in politica interna alcuni problemi tornano alla ribalta alla fine del 2000. L'inflazione infatti torna a salire ed i salari, stabilizzati nel triennio precedente, tornano a scendere. All'inizio del 2001 il tracollo della compagnia aerea nazionale privatizzata scuote bruscamente l'economia bulgara. L'impatto delle riforme economiche si rivela molto serio per chi dispone di redditi fissi, pensionati e genitori non sposati, la disoccupazione raggiunge il $18 \%$. I giovani, soprattutto, sono colpiti da questa nuova ondata di crisi economica, senza contare i dati allarmanti sul crimine, mai del tutto debellato secondo i dettami dell'Unione Europea. Nel maggio del 2000 si calcola che il fatturato delle attività illegali ammonta a un terzo del PIL nazionale, questa la vera piaga della Bulgaria contemporanea. I governi che si sono succeduti dal 1989 in poi non hanno saputo porre rimedio ad una delle pressanti richieste di Bruxelles. II governo Kostov perde quindi la sua battaglia: all'inizio del nuovo secolo la corruzione è ormai l'elemento caratterizzante della scena politica bulgara. In

${ }^{7}$ Nella primavera del 1999, per circa 80 giorni, il territorio jugoslavo è stato teatro di un conflitto atipico. Per la prima volta nella storia militare, una massiccia campagna aerea ha risolto una situazione di crisi internazionale senza che fosse necessario ricorrere a un intervento terrestre. Una guerra particolare, che ha visto contrapposte la NATO, al suo primo impegno offensivo in cinquant'anni di esistenza e la Federazione Serbo-Montenegrina, trascinata verso il baratro di una sconfitta, sia pur parziale, dalla cinica politica del suo presidente Milosevic. Per uno sguardo d'insieme e l'analisi delle operazioni belliche, il dettaglio delle forze contrapposte, l'esame tecnico dei mezzi aerei, di quelli navali e dei sistemi d'arma. 
giugno l'episodio più nefasto. II responsabile del governo per le trattative con l'UE, Aleksandar Bozkov, è costretto a lasciare il suo posto dopo che la Procura generale ne denuncia le attività a Kostov. La vicenda che però tracima la pazienza dell'opinione pubblica ha luogo in settembre, quando il quotidiano dell'opposizione, "Trud", rivela che la mafia russa aveva fornito 80 mila dollari a una fondazione filantropica alla cui guida c'è la moglie del primo ministro, Elena Kostova. La reputazione dell'esecutivo ne risente in maniera irrimediabile, ovviamente. All'inizio del 2001 l'opinione pubblica bulgara s'interroga riguardo alla possibile corsa alle imminenti elezioni dell'ex sovrano Simeone II, in esilio da anni. La sua candidatura, secondo la Costituzione, sarebbe stata impossibile poiché le leggi dello Stato richiedono che ogni aspirante alla presidenza fosse residente in Bulgaria da almeno cinque anni. Simeone torna a Sofia in aprile, certamente fuori "tempo costituzionale", e annuncia la nascita di una nuova formazione politica, Movimento nazionale Simeone II ( NDSV), ed il chiaro intento di prendere parte alle elezioni parlamentari. II programma del nuovo partito dell'ex re Simeone II è subito definito populista dall'opinione pubblica bulgara, in estrema sintesi si prefigge di porre fine al settarismo politico, di eliminare la corruzione e una riforma qualitativa che avrebbe introdotto un'economia di mercato in linea con i requisiti richiesti dall'Unione Europea. II target di riferimento del nuovo movimento politico bulgaro sono i giovani e le donne, difatti, a dimostrazione di ciò, Simeone II si circonda di collaboratori giovani e di numerose donne dalle più svariate esperienze professionali. L'NDSV promette inoltre un drastico aumento delle pensioni, degli assegni familiari e dei salari, piuttosto bassi all'epoca, degli insegnanti. Tocca le corde giuste, almeno sembra. II programma di Simeone II è molto semplice nella sua costruzione tipica, introduce elementi nuovi e sicuramente di forte impatto popolare. Nei sondaggi schizza subito al primo posto e conferma la buona campagna elettorale, gestita a suon di promesse, confermando la posizione alle elezioni del 17 giugno, quando si assicura il 42\% delle preferenze e ben 120 seggi parlamentari, la metà del totale. Un altro dato politico molto interessante riguarda il record, stabilito dal voto del 2001, del più alto numero di deputati donne della storia bulgara. II movimento, oltre a raccogliere i favori di giovani, pensionati e dell'elettorato femminile, ottiene il voto dei turchi di Bulgaria, sia in patria sia tra gli emigrati in Turchia (Motta, 1998), che nel 1994 avevano partecipato alle elezioni solo in 2mila, nel 2001 toccano quota 55mila. Simeone, ad ogni modo, è dotato di fascino personale, riconosciuto in patria ma anche all'estero e, requisito assai importante, non aveva rapporti con nessuno dei due gruppi che avevano monopolizzato la scena politica dopo la caduta di Zivkov. Un personaggio "borderline" in un contesto politico incapace di evolversi. Si apre il "governo del re" e la strada verso la NATO e l'Unione europea inizia a presentarsi non più in salita. L'impresa di Simeone II è comunque notevole, difficile infatti appariva per un ex sovrano tornare nel proprio Paese d'origine, un tempo comunista, nelle vesti di primo ministro ma, cosa assai più complicata, ottenere successo malgrado le problematiche interne appariva ai più un'impresa quasi impossibile. Dopo il suo rientro in Bulgaria Simeone adotta il cognome ufficiale di Sakskoburggosski ${ }^{8}$, sebbene tutti lo chiamano carja, il re. II suo ministro degli Esteri, Solomon Passy, appartiene alla comunità ebraica bulgara ed è sostenitore dell'adesione del Paese alla NATO. Le figure principale dell'esecutivo sono due giovani tecnocrati della finanza: Nikolai Vasilev, trentunenne nominato ministro dell'Economia e vice primo ministro, e Milen Velcev, trentacinquenne che ricopre la carica di ministro delle Finanze. I principali obiettivi del nuovo governo sono rappresentati dal miglioramento dei requisiti d'ingresso nell'Unione europea, adesione alla NATO, accelerazione della riforma economica, miglioramento della qualità della vita, contenimento del crimine e ovviamente lotta alla corruzione. L'esecutivo decide di dare preminenza alla realizzazione del piano relativo le riforme economiche. II 2 agosto il ministro Vasilev promette che il mercato dell'energia sarebbe stato liberalizzato e che la privatizzazione delle risorse principali, come le centrali elettriche, sarebbe stata accelerata. In ottobre viene così creato un fondo di 20 milioni di dollari di lev che deve fornire microcrediti alle piccole imprese. Sul fronte sociale si stabilisce che dal $1^{\circ}$ ottobre il salario minimo sarebbe stato di 100 lev, circa 50 dollari. Aumentati contestualmente anche gli stipendi dei dipendenti pubblici del 17\%, mentre dal $1^{\circ}$ gennaio 2002 sarebbero raddoppiati gli assegni familiari. Sempre nell'ottobre 2001 arriva però una notizia inattesa per il nuovo esecutivo. I vertici comunitari, infatti, stabiliscono che la Bulgaria non soddisfa ancora pienamente la condizione sine qua non per l'ammissione all'UE, non è una "reale economia di mercato". Difatti alla conferenza di Laeken, del dicembre 2001, Sofia non avrebbe partecipato nella lista del primo gruppo di Stati che sarebbero rientrati nel processo di allargamento. II governo di Sakskoburggotski incontra sempre maggiori difficoltà a convincere il Paese della necessità di convenire alle richieste di Bruxelles. Nel novembre del 2001 le elezioni presidenziali sono vinte da Gerogi Parvanov, leader del BSP. Durante l'autunno del 2001, inoltre, Bruxelles preme affinché fosse affrontata in maniera chiara e decisa la questione dei rom. II governo accelera l'introduzione di un programma di sostegno all'inserimento dei rom nella società, già abbozzato dal governo Kostov nel 1999. II PIL, ad ogni modo, continua a crescere stabilizzando l'economia di Sofia. Nel 2002 il rating creditizio della Bulgaria è alzato per cinque volte e a Milen Velcev è assegnato il premio Euromoney di ministro delle Finanze più virtuoso dell'anno. Gli sforzi del governo sembrano avere successo. Nel

${ }^{8}$ Casa nobiliare tedesca di Sassonia - Coburgo - Gotha. II nome deriva dal Ducato di Sassonia - Coburgo - Gotha. 
dicembre 2002 la conferenza europea di Copenaghen stabilisce che i negoziati per l'entrata della Bulgaria e della Romania nella comunità sarebbero dovuti partire entro il $1^{\circ}$ gennaio 2007. Un grande passo in avanti verso l'ammissione si raggiunge nel novembre 2003, allorché l'UE annuncia che la Bulgaria aveva raggiunto lo status di "reale economia di mercato", una grandissima soddisfazione per l'esecutivo ed il portentoso ministro dell'Economia Milen Velcev. L'obiettivo sembra più vicino che mai. Ulteriori progressi sono registrati durante il 2004 quando il parlamento europeo rivolge un incoraggiante messaggio alla Bulgaria. II 14 giugno dello stesso anno, in Lussemburgo, sono dichiarate concluse le trattative per l'ingresso di Sofia nell'UE, soddisfatti quindi i 26 capitoli dell'acquis comunitario. La strada d'ingresso nella NATO si rivela più facile del previsto. L'utilizzo di una base da parte delle aerocisterne KC - 135 Stratotanker americane, durante la guerra in Afghanistan del 2001, fa compiere un balzo in avanti nella trattativa. Un settore in crescente sviluppo, quello militare, vedeva intanto la trasformazione del suo esercito di leva in un'armata professionale, con un occhio ai costi. Nel novembre 2003 la NATO si esprime positivamente riguardo le riforme del governo di Sofia e durante una conferenza tenuta a Praga, la Bulgaria è invitata a entrare nell'alleanza. Inoltre, dopo la guerra in Iraq, Sofia invia le sue truppe nel Paese mediorientale schierando circa cinquecento soldati nella città di Karbala, sotto comando polacco. Ad aprile del 2004 la Bulgaria entra a pieno titolo nell'alleanza atlantica. Le questioni di politica interna più importanti, lotta alla corruzione e al crimine organizzato, sono anch'esse portate avanti dal neo governo del re che annuncia una strategia nazionale anticorruzione. Un piano d'azione che prevede l'istituzione di una direzione nazionale per la lotta al crimine organizzato, parallelamente all'aiuto ed alla consulenza di Scotland Yard per prevenire e stanare la corruzione all'interno delle istituzioni governative e di polizia nazionale. Nel 2003 circa 200 funzionari sono licenziati e altri 800 sono messi sotto inchiesta, il piano sembrava sortire i suoi effetti. La percezione dei successi, in campo economico e amministrativo, del nuovo governo, da parte dei cittadini, risultano poco evidenti, poiché ai progressi macroeconomici bisogna affiancare le difficoltà sul piano microeconomico. Nel suo rapporto annuale, ad esempio, l'Agenzia statunitense per lo sviluppo internazionale, USAID, afferma che il cittadino bulgaro è afflitto dalla povertà, dalla disoccupazione e da un tenore di vita scadente. Bisognava poi tenere conto del fenomeno della emigrazione, che dopo il crollo del comunismo priva la nazione bulgara di circa 700mila persone, molti dei quali giovani. L'opinione pubblica inizia perciò a ravvisare i primi malumori, mai sopiti in realtà dopo il 1989, delle classi medio - basse. Disagio determinato dagli alti redditi di un gruppo esiguo della popolazione e dalle scarse condizioni di vita della maggioranza dei cittadini bulgari. Questo, tra i tanti, uno dei problemi da risolvere all'indomani dell'ingresso della Bulgaria, $1^{\circ}$ gennaio 2007, come Paese membro dell'Unione europea.

\section{Conclusioni}

L'era del "post-totalitarismo" ha disatteso le speranze di un veloce progresso materiale e le numerose riforme economiche hanno comportato grossi costi sul piano sociale. E' sintomatico che, dopo il 1989, per oltre cinque anni i governi di Bulgaria non siano stati capaci di accordarsi sulla scelta del nuovo simbolo nazionale. Sulla poca certezza della loro posizione in ambito internazionale e sulle insicurezze interne sono stati scritti numerosi articoli e volumi in Europa. Tutti i lavori cercano di interpretare "l'essere bulgari", la "bulgarità". La storia certamente può aiutare la Bulgaria. Uno dei temi prediletti della storia bulgara si sostanzia, ad esempio, nella più volte citata, dagli studiosi, dicotomia tra una propensione all'Oriente e una all'Occidente, come nel caso della cristianità. Nei secoli scorsi una piccola frangia nazionalista vagliava l'opportunità di affidarsi alla protezione russa o a quella dell'Europa centro - occidentale. In campo strettamente politico la decisione di schierarsi con o contro la Russia non poteva certamente non portare conseguenze di un certo rilievo. Nel 1913 e nel 1915, così come nel 1941, Sofia scelse di contrastare gli interessi russi, ottenendo pessimi risultati che rallentarono le proprie aspirazioni nazionali. Dopo il 1944 i politici bulgari tornarono sui loro passi, verso un rapporto più stretto con la Russia. Nel periodo immediatamente successivo al 1989, la politica estera bulgara cambia nuovamente rotta, avvicinandosi all'Unione europea, come abbiamo visto. Crescono inoltre i sentimenti filoamericani ed europeisti. A causa delle sanzioni economiche stabilite contro il Montenegro e la Serbia, il corso degli eventi ha fatto si che la Bulgaria si trovasse fuori dall'area dell'Europa centrale e occidentale, proprio nel momento di maggiore sforzo politico teso all'integrazione con i Paesi di quelle aree. La Bulgaria si è poi riavvicinata al mondo occidentale durante le due guerre statunitensi in Afghanistan e in Iraq. Nell'estate del 2004 si è aperta per Sofia una nuova fase di integrazione nell'Europa centrale e occidentale. II Paese, ad ogni modo, oscillerà sempre, secondo le previsioni di autorevoli studiosi, tra la sua vocazione orientale e quella occidentale per via della sua posizione geografica. Crocevia di comunicazione tra due continenti, Bulgaria terra di confine. Molti sono ad esempio i progetti che in questi anni sono al vaglio delle amministrazioni pubbliche bulgare, in collaborazione con i Paesi confinanti. Progetti di respiro edilizio che prospettano nuove vie di comunicazione, nuove autostrade tra Durazzo e Istanbul attraverso la Macedonia ed 
appunto la Bulgaria, riprendendo il vecchio tracciato della Via Egnatia di memoria romana. Dopo più di vent'anni si può inoltre affermare che la stabilità politica è stata raggiunta. Analizzando il periodo successivo al 1989 si osserva un rispetto della volontà popolare, importante punto di partenza e di salvaguardia in una vera democrazia. Sette elezioni politiche e quattro elezioni presidenziali. Sofia in questi anni ha sperimentato un governo di minoranza del BSP, un governo dello SDS, due governi tecnici non partitici, due governi del BSP con maggioranza parlamentare ed un governo dell'ex sovrano. Allo stesso tempo si è posto il massimo rispetto alle libertà individuali e si è tentato di migliorare la condizione delle minoranze etniche. Certo il crimine e la corruzione rimangono problemi ancora da risolvere. Sia il governo Kostov, come abbiamo detto, che quello del re hanno combattuto senza riserva queste due criticità. La strada verso una sana democrazia è stata però intrapresa e difficilmente verrà abbandonata.

\section{Bibliography}

AA. VV. (2011), Turchia, un attore mediterraneo ed internazionale, Roma, Alpes Italia.

Aslanian D. (2004), Storia della Bulgaria dall'antichità ai giorni nostri, Milano, Trimontium.

Arielli E. (1999), La guerra del Kosovo. Anatomia di un'escalation, Roma, Editori Riuniti.

Barker E. (1950), Macedonia: It's Place in the Balkan Power Politics, London.

Bell J.D. (1986), The Communist Party of Bulgaria since Blagoev to Zhivkov, Stanford.

Biagini A. (1981), Momenti di Storia Balcanica (1878-1914), Roma.

Biagini A. (1990), L'evoluzione del Paesi dell'Est dal 1945 ad oggi, in "La grande Europa, la nuova Europa: opportunità e rischi", Rocca Salimbeni.

Biagini A., F. Guida (1994), Mezzo secolo di socialismo reale: l'Europa orientale dal 1945 ad oggi, Torino, Giappichelli.

Biagini A. (2005), Storia della Turchia contemporanea, Milano, Bompiani.

Biagini A. (2006), Storia dell'Ungheria contemporanea, Milano, Bompiani.

Bozzo L.(2000), Macedonia: la nazione che non c'è. Una fragile democrazia tra conflitto etnico e crisi regionale, Roma, FrancoAngeli.

Brailsford H.N. (1986), Macedonia: its Races and their Future, London.

Bruno F. (2012), Stati membri e Unione europea. II difficile cammino dell'integrazione, Torino, Giappichelli.

Brown J.F. (1970), Bulgaria under Communist Rule, London.

Bucar V. (1979), La Macedonia e i macedoni. Cenni di storia, politica e cultura, Olshky.

Crampton R.J. (2010), Bulgaria. Crocevia di culture, Trieste, Beit Storia.

Dahrendorf R. (1999), 1989. Riflessioni sulla rivoluzione in Europa. Lettera immaginaria ad un amico di Varsavia, Bari, Laterza.

D’Amato G. (2008), L'eurosogno ed i nuovi muri ad est. L'Unione europea e la dimensione orientale, Roma, Nagre.

Dimitrov V. (2011), Bulgaria: the Uneven Transition, London.

Fatutta F. e Peruzzi L. (2000), L'eurosogno ed i nuovi muri ad est. L'Unione europea e la dimensione orientale, Milano, Mursia Editore.

Giatzidis E. (2002), An introduction to Post - Communist Bulgaria, Manchester, Manchester University Press.

Gokay B. (2005), L'Europa orientale dal 1970 ad oggi, Bologna, II Mulino.

Graciotti S. (2007), S. Chiese ortodosse e Chiesa cattolica nel balcano slavo sottomesso all'Islam ottomano, Europa Orientalis, XXVI.

Isusov M. (1975), Problems of Transition form Capitalism to Socialism, Sofia.

Lampe J.R. (1986), The Bulgarian Economy in the Twentieth Century, London.

Leonardi S.(1977), L'Europa e il movimento socialista; Considerazioni sui processi comunitari: CEE e Comecon, Milano, Aldelphi.

Magnoli A. (2007), La Banca Mondiale, Bologna, II Mulino.

McIntryre R.J. (1988), Bulgarian: Politics, Economics and Society, London.

Moser C.A. (1989), Dimitrov of Bulgaria: A Political Biography of Dr Georgi D. Dimitrov, Ottawa.

Motta G. (1998), I Turchi, il Mediterraneo, l'Europa, Milano, FrancoAngeli.

Pandev M. (1948), Dimitroff Wastes No Bullets: the Inside History of the Trial and Murder of Nikola Petkov, London.

Perich A. (1998), Origine e fine della Jugoslavia, Bologna, Lupetti Editore.

Ramet S.P. (2009), Serbia, Croatia and Slovenia at Peace and at War, London, Central Books.

Schlitzer G. (2011), II Fondo monetario internazionale, Bologna, II Mulino.

Simsir B.N. (1985), The Turks of Bulgaria (1878-1985), Maastricht.

Trinchese S. (2005), Mare Nostrum. Percezione Ottomana e Mito Mediterraneo in Italia all'Alba del '900, Milano. 